\title{
The effect of a laminar moving flame front on thermoacoustic oscillations of an anchored ducted V-flame
}

\author{
Charles M. Luzzato * Aimee S. Morgans ${ }^{\dagger}$
}

October 6, 2014

\begin{abstract}
When investigating combustion instabilities using analytical models, it has previously been assumed that the compact flame assumption implied that the flame-front movement did not need to be taken into account to solve the acoustics. This paper shows that this is not necessarily the case. This paper presents a generalisation of such models of anchored V-flames to allow the flame "source" of acoustic waves to vary its position in time so as to track the flame-front location. A method for solving this problem is then presented. It is found that accounting for the flame front movement can alter both the linear stability of the combustor, and (for cases that remain unstable) the limit cycle amplitude. Significant changes in limit cycle amplitude are observed across a large range of operating conditions. The flame front movement has so far only been seen to provide a stabilising effect, reducing the Rayleigh source term. Self-Tuning Regulator adaptive control methods appear to be unaffected by accounting for the moving flame front.
\end{abstract}

\section{Introduction}

The drive to reduce $\mathrm{NO}_{x}$ emissions from aircraft and land based gas plants has motivated the use of leaner combustion in turbine engines. This has led to the design of lean premixed combustors, where the fuel and air are premixed upstream of the combustor chamber, and the fuel to air concentration is small compared to stoichiometric conditions. In lean premixed combustion, the flame and heat release are very sensitive to external perturbations (Candel, 2002; Lieuwen, 2003). In certain cases, this can induce self sustained combustion instabilities driven by the two-way interaction of combustor acoustics and unsteady heat release (Rayleigh, 1878). These combustion instabilities lead to an

\footnotetext{
${ }^{*} \mathrm{PhD}$ student, Department of Aeronautics, Imperial College.
}

${ }^{\dagger}$ Senior Lecturer, Department of Aeronautics, Imperial College 
increase in noise and can have an important impact on the structural behaviour of the combustor. Understanding, preventing and suppressing these instabilities is therefore a research priority.

In order to model combustion instabilities, it is necessary to combine an acoustic model and a flame model. The former captures the generation of acoustic waves by unsteady heat release and their subsequent behaviour within the combustor, whilst the latter models the response of the unsteady heat release rate to acoustic perturbations. It has been widely shown that linear acoustic models suffice (Peracchio and W. M. Proscia, 1999; Noiray et al., 2008), even in limit cycle. Acoustic models which are based on either Galerkin basis expansions (Culick, 1988) or a wave description (Dowling and Stow, 2003) can be used, the latter having the advantage of allowing many types of acoustic boundary condition and extending naturally to azimuthal as well as plane waves in annular combustor geometries (Morgans and Stow, 2007; Stow and Dowling, 2009). The flame model must be non-linear in order to capture saturation into limit cycle for unstable combustors (Peracchio and W. M. Proscia, 1999; Noiray et al., 2008), and is therefore more challenging. Much work has been done to understand the response of the flame and heat release to perturbations in both laminar and turbulent flames (Candel, 2002; Lieuwen, 2003; Preetham and Lieuwen, 2007; Hemchandra et al., 2011). Recent examples include showing that variations in laminar burning velocity due to flame stretch and curvature can induce decay of flame wrinkling for high Strouhal numbers (Preetham et al., 2010), and that accounting for the effects of phase velocity near the flame can alter the flame describing function and hence the limit cycle amplitude (Preetham et al., 2008; Kashinath et al., 2013). Even though limit cycle oscillations are the most widely observed non-linear final state, it has recently been found that other non-linear final states, such as chaotic behaviour, are also possible experimentally (Kabiraj and Sujith, 2012).

Due to the complexity of state-of-the-art combustor models, fundamental investigations are frequently performed on simplified combustor models in the first instance (Dowling, 1999; Balasubramanian and Sujith, 2008; Hield et al., 2009; Goh and Morgans, 2013). Simple combustor models of an 'anchored ducted laminar flame' which use the G-Equation flame model (Fleifil et al., 1996; Dowling, 1999; Schuller et al., 2003; Blumenthal et al., 2013) have been particularly popular. The G-equation models the kinematics of the flame non-linearly, capturing effects such as saturation into limit cycle, and showing reasonable agreement with experimental results (Langhorne, 1988a; Evesque, 2000; Schuller et al., 2003).

When implementing the G-Equation in anchored ducted-flame models, it has always previously been 
assumed that the discontinuity or "jump" in the acoustic wave amplitude due the flame remains immobile and at the flame anchor position. This is despite the fact that the time-space average position of the flame is generally located a distance downstream of the anchor, and that furthermore the instantaneous spatially averaged position of the flame oscillates in time. Some work has already been done to take into account a varying time delay required for a perturbation to reach the flame (Yuan et al., 2010), but these do not include the effects of a moving acoustic discontinuity.

In the compact assumption, the stream-wise extent of the flame as well as the extent of its motion are assumed small compared to the wavelength of the acoustic modes. Other works have investigated the validity of assuming that the streamwise extent of the flame is small (Rayleigh, 1878; Noiray et al., 2008). In this work we investigate the effect of the flame motion, assuming that at any moment in time the flame itself is compact. Whilst it seems intuitive that the large flame oscillations associated with limit cycle oscillations (ensuing from linear instability) are likely to induce a dependence on the flame motion, we also explain that the time-variation can affect linear stability itself. Thus we perform an investigation into the effect of a time-varying flame front location on thermoacoustic oscillations, and on the Rayleigh source term that drives them. For simplicity, we neglect hydrodynamic effects and any turbulent perturbations; this means that the flame-front cannot be multi-valued (Shin and Lieuwen, 2013), thus allowing the kinematic flame front tracking version of the G-equation to be used.

In order to do this, we derive the expressions governing the spatially-averaged flame location (which varies with time), and we then allow the position of the discontinuity in the acoustic wave amplitude to track this in time. This enables us to compare the thermoacoustic behaviour under three different assumptions:

- The flame-induced discontinuity in acoustic wave amplitude is fixed at the flame anchoring point (i.e. the standard implementation).

- The flame-induced discontinuity in acoustic wave amplitudes is fixed at the space-averaged steady flame position.

- The flame-induced discontinuity in acoustic wave amplitudes moves in time to match the spatial-mean of the heat release location.

This allows us to deduce whether the shift in the time-mean position of the discontinuity, or its time-variation, are responsible for changes in the thermoacoustic behaviour. 


\section{Review of the anchored ducted-flame model}

We first review the anchored ducted V-flame model in the form in which it has previously been used. The model considers (i) the acoustic waves and (ii) the flame motion, coupling these via equations for the "jumps" or discontinuities in acoustic variables across the flame anchor position.

Considering first the acoustic waves, the steady mean flow is considered uniform upstream and downstream of the flame, and vorticity and entropy waves are neglected. The flow variables can be decomposed into a steady mean, and a small acoustic fluctuation: $(p(x, t), u(x, t), \rho(x, t))=$ $(\bar{P}, \bar{U}, \bar{\rho})+\left(p_{a}(x, t), u_{a}(x, t), \rho_{a}(x, t)\right)$, such that only linear fluctuations need be retained. Frequencies are assumed sufficiently low for only plane acoustic waves to exist (Dowling and Williams, 1983), and the linearised acoustic equations are obtained as:

$$
\begin{aligned}
\frac{1}{\bar{c}^{2}} \frac{\partial p_{a}}{\partial t}+\bar{\rho} \frac{\partial u_{a}}{\partial x}+\frac{\bar{U}}{\bar{c}^{2}} \frac{\partial p_{a}}{\partial x} & =0 \\
\bar{\rho}\left(\frac{\partial u_{a}}{\partial t}+\bar{U} \frac{\partial u_{a}}{\partial x}\right) & =-\frac{\partial p_{a}}{\partial x}
\end{aligned}
$$

where the density is $\rho_{a}(x, t)=\frac{p_{a}(x, t)}{\bar{c}^{2}}$ and $\bar{c}$ is the speed of sound.

The acoustic pressure $p_{a}$, acoustic velocity $u_{a}$, and acoustic density $\rho_{a}$ can then be solved analytically using the method of characteristics (Rienstra and Hirschberg, 2012); with upstream and downstream travelling acoustic waves either side of the "flame", as shown in Figure 1.

[Figure 1 about here.]

Upstream of the flame-induced discontinuity (indicated by subscript ${ }_{u}$ ) the acoustic variables are (Dowling, 1997):

$$
\begin{aligned}
& p_{a}(x, t)=C\left(t-\tau_{u_{C}}(x)\right)+A\left(t+\tau_{u_{A}}(x)\right) \\
& u_{a}(x, t)=\frac{C\left(t-\tau_{u_{C}}(x)\right)-A\left(t+\tau_{u_{A}}(x)\right)}{\bar{\rho}_{u} \bar{c}_{u}} \\
& \rho_{a}(x, t)=\frac{C\left(t-\tau_{u_{C}}(x)\right)+A\left(t+\tau_{u_{A}}(x)\right)}{\bar{c}_{u}^{2}}
\end{aligned}
$$


while downstream (indicated by subscript ${ }_{d}$ ):

$$
\begin{aligned}
& p_{a}(x, t)=B\left(t-\tau_{d_{B}}(x)\right)+D\left(t+\tau_{d_{D}}(x)\right) \\
& u_{a}(x, t)=\frac{B\left(t-\tau_{d_{B}}(x)\right)-D\left(t+\tau_{d_{D}}(x)\right)}{\bar{\rho}_{d} \bar{c}_{d}} \\
& \rho_{a}(x, t)=\frac{B\left(t-\tau_{d_{B}}(x)\right)+D\left(t+\tau_{d_{D}}(x)\right)}{\bar{c}_{d}^{2}}
\end{aligned}
$$

The time delays

$$
\begin{aligned}
\tau_{u_{A}}(x)=\frac{x-x_{b}}{\bar{c}_{u}-\bar{U}_{u}} & \tau_{u_{C}}(x)=\frac{x-x_{b}}{\bar{c}_{u}+\bar{U}_{u}} \\
\tau_{d_{B}}(x)=\frac{x-x_{b}}{\bar{c}_{d}+\bar{U}_{d}} & \tau_{d_{D}}(x)=\frac{x-x_{b}}{\bar{c}_{d}-\bar{U}_{d}}
\end{aligned}
$$

represent:

- $\tau_{u_{A}}(x)$ : the time taken for wave $A$ to go from the discontinuity $x_{b}$ to a point upstream $x$;

- $\tau_{u_{C}}(x)$ : the time taken for wave $C$ to go from a point upstream $x$ to the discontinuity $x_{b}$;

- $\tau_{d_{B}}(x)$ : the time taken for wave $B$ to go from the discontinuity $x_{b}$ to a point downstream $x$;

- $\tau_{d_{D}}(x)$ : the time taken for wave $D$ to go from a point downstream $x$ to the discontinuity $x_{b}$.

Pressure reflection coefficients are used to impose physical behaviour at boundaries:

$$
\begin{aligned}
& C\left(t-\tau_{u_{C}}\left(x_{u}\right)\right)=R_{u} A\left(t+\tau_{u_{A}}\left(x_{u}\right)\right) \\
& D\left(t+\tau_{d_{D}}\left(x_{d}\right)\right)=R_{d} B\left(t-\tau_{d_{B}}\left(x_{d}\right)\right)
\end{aligned}
$$

After a simple shift in time we obtain:

$$
\begin{aligned}
& C(t)=R_{u} A\left(t-\tau_{u}\right) \\
& D(t)=R_{d} B\left(t-\tau_{d}\right)
\end{aligned}
$$

where $\tau_{u}=-\tau_{u_{A}}\left(x_{u}\right)-\tau_{u_{C}}\left(x_{u}\right)$ and $\tau_{d}=\tau_{d_{B}}\left(x_{d}\right)+\tau_{d_{D}}\left(x_{d}\right)$.

The effects of the flame on the acoustics are imposed at the flame anchoring position. Wave amplitudes either side of the the anchor position are related by imposing the flow conservation equations across the flame (Dowling, 1997). The conservation of energy means that the acoustic wave amplitudes are not constant across the flame, but rather experience a "jump" or discontinuity, which 
depends upon the total heat release rate, $Q$, of the flame (Dowling, 1995, 1997). The non-linear form of the jumps is given as:

$$
\begin{aligned}
\rho_{u} u_{u}[u]+[p] & =0 \\
\frac{\gamma}{(\gamma-1)}[u p]+\frac{1}{2} \rho_{u} u_{u}\left[u^{2}\right] & =\frac{Q}{\mathcal{A}_{d u c t}}
\end{aligned}
$$

where we use the continuity equation to write $[\rho u]=\rho\left(0_{d}, t\right) u\left(0_{d}, t\right)-\rho\left(0_{u}, t\right) u\left(0_{u}, t\right)=0$, and all variables are evaluated at $x=0$. We note that $\gamma$ is the specific heat capacity, and $\mathcal{A}_{\text {duct }}$ the area of the duct cross-section. Linearising these equations, and using the fact that the above equations are satisfied by the steady flow yields:

$$
\begin{aligned}
\bar{\rho}_{u} \bar{U}_{u}\left[u_{a}\right]+\left[p_{a}\right]+\left(\bar{\rho}_{u} u_{a_{u}}+\bar{U}_{u} \rho_{a_{u}}\right)[\bar{U}] & =0 \\
\frac{\gamma}{(\gamma-1)}\left(\left[\bar{U} p_{a}\right]+\left[\bar{P} u_{a}\right]\right)+\bar{\rho}_{u} \bar{U}_{u}\left[u_{a} \bar{U}\right]+\frac{1}{2}\left(\bar{\rho}_{u} u_{a_{u}}+\rho_{a} \bar{U}_{u}\right)\left[\bar{U}^{2}\right] & =\frac{Q^{\prime}}{\mathcal{A}_{d u c t}}
\end{aligned}
$$

A flame model is needed to capture how the variation in $Q(t)$ depends on the incoming acoustic excitation. The well-known G-Equation model assumes that the flame responds to the flow velocity directly upstream, its motion being governed by a non-linear partial differential equation: the GEquation. This is derived by assuming that the flame initiation surface, $\mathcal{G}$, is convected by its relative burning velocity $u_{\text {gutter }}-S_{u} / \mathbf{n}$ (n being the unit normal to the flame), such that $\frac{D \mathcal{G}}{D t}=0$ (Kerstein et al., 1988). The movement and shape of the flame are then tracked by considering $\mathcal{G}=x-\xi(r, t)$ (Fleifil et al., 1996; Dowling, 1999); this yield the simpler flame-front tracking version of the G-Equation:

$$
\frac{\partial \xi}{\partial t}=u_{\text {gutter }}-S_{u} \sqrt{1+\left(\frac{\partial \xi}{\partial r}\right)^{2}}
$$

where $u_{\text {gutter }}$ is the velocity just upstream of the discontinuity, $r$ is the radial position in the duct, and $S_{u}$ is the laminar burning velocity, usually chosen empirically. For one dimensional flow, the velocity $u_{\text {gutter }}$ is given by the sum of steady and fluctuating acoustic velocity, $u_{\text {gutter }}=\bar{U}_{u}+u_{a}\left(0_{u}, t\right)$. Anchoring of the flame is imposed through $\xi=0$ at the anchor point (here $x=x_{b}=0$ ).

The steady state of the flame is obtained by writing:

$$
\bar{U}_{u}-S_{u} \sqrt{1+\left(\frac{\partial \bar{\xi}}{\partial r}\right)^{2}}=0
$$


which leads to the steady solution

$$
\frac{\partial \bar{\xi}}{\partial r}= \pm \sqrt{\left(\frac{\bar{U}_{u}}{S_{u}}\right)^{2}-1}
$$

The full steady shape of the flame is easily obtained from (20) and the anchoring boundary condition. It is assumed that $Q(t) \propto \mathcal{A}\left(t-\tau_{f}\right)$ (Dowling, 1999; Wang and Dowling, 2005; Preetham et al., 2010) where $\tau_{f}$ is chosen empirically as $\tau_{f}=\frac{0.42\left(x_{d}-x_{b}\right)}{u_{g u t t e r}}$ and the flame area $\mathcal{A}$ is given by:

$$
\mathcal{A}(t)=\int_{r_{a}}^{r_{b}} 2 \pi R \sqrt{1+\left(\left.\frac{\partial \xi}{\partial r}\right|_{r=R}\right)^{2}} d R
$$

where $r_{a}$ is the radius of the flame holder, and $r_{b}$ is the radius of the duct. Thus at any point in time, the jump in the wave amplitude either side of the flame depends on the flame area and hence the instantaneous flame shape.

\section{Time-varying systems: effect on linear stability}

Although the flame moves in the duct around its anchoring point in the ducted flame model described above, the location at which the resulting discontinuity in the acoustic wave strengths occurs is assumed fixed in time. If the movement of this discontinuity was accounted for, it would become time-varying; the acoustic time-delays relevant to the flame would then also be time varying, as shown in Figure 2. The effect of this on the ducted-flame model will be investigated later. First however, it is instructive to consider the effect that time-variation can have on linear stability. It seems intuitive that during limit-cycle, when the flame oscillations are large, non-linear time-varying effects are likely to be important. However, time-variation can also affect linear stability, as shown in the illustrative example below.

[Figure 2 about here.]

Consider the one degree of freedom system represented by a second order differential equation with a time varying coefficient, as shown in equation (22). This is known as the Mathieu equation, and the analysis of its stability is the subject of Floquet Theory (Floquet, 1883; Bessa, 2012; Ghose Choudhury and Guha, 2014). 


$$
\frac{\partial^{2} u}{\partial t^{2}}+\left(\bar{a}+a^{\prime}\right) u(t)=0
$$

The stability diagram of the above Mathieu equation is shown in Figure 3, having been calculated using the method of Hill's determinant. The curve shows the frontier between stability and instability, with the stable region lying below the curve. The stable region is a function of $\bar{a}$ and $a^{\prime}$; as the amplitude of oscillations increases, the stable region becomes smaller, and new unstable regions appear. This serves to illustrate that time variation can affect linear stability at small amplitudes, as well as being influential under large amplitude oscillations.

[Figure 3 about here.]

\section{Varying the discontinuity position in the G-Equation ducted flame model}

In section 2, the position at which the flow conservation equations (16) and (17) were applied and at which the acoustic waves experienced a discontinuity $\left(x_{b}\right)$, was fixed in time at the flame anchoring position, $x_{b}=0$. For compact flames and small amplitude oscillations, this is usually a good approximation (Dowling, 1997). However, when the combustor is unstable, the amplitude of the flame motion can become large (Langhorne, 1988b). Then, even under the simplifying assumption that the heat release occurs at a single axial location (necessary when assuming plane acoustic waves), $x_{b}(t)$, the flame-induced acoustic wave discontinuity should track the spatially-averaged flame position $x_{f}(t)$.

In order to track $x_{f}(t)$, and allow a time variation in the acoustic wave discontinuity, the time delays (8) and (9) become:

$$
\begin{array}{rlr}
\tau_{u_{A}}(x, t)=\frac{x-x_{b}(t)}{\bar{c}_{u}-\bar{U}_{u}} & \tau_{u_{C}}(x, t)=\frac{x-x_{b}(t)}{\bar{c}_{u}+\bar{U}_{u}} \\
\tau_{d_{B}}(x, t)=\frac{x-x_{b}(t)}{\bar{c}_{d}+\bar{U}_{d}} & \tau_{d_{D}}(x, t)=\frac{x-x_{b}(t)}{\bar{c}_{d}-\bar{U}_{d}}
\end{array}
$$

where $x_{b}(t)$ tracks the average location of the heat release, $x_{f}(t)$, in time, i.e. $x_{b}(t)=x_{f}(t) . x_{f}(t)$ 
is given by the area-weighted mean flame position $\left(\right.$ as $\left.Q(t) \propto \mathcal{A}\left(t-\tau_{f}\right)\right)$ :

$$
x_{b}(t)=x_{f}(t)=\frac{\sum_{k=1}^{n} \mathcal{A}_{k}\left(t-\tau_{f}\right) \xi_{k}\left(t-\tau_{f}\right)}{\sum_{k=1}^{n} \mathcal{A}_{k}\left(t-\tau_{f}\right)}
$$

where each subscript ${ }_{k}$ denotes a section of the flame cut along $r$. As such, $\mathcal{A}_{k}$ is the incremental flame area at the discrete position $\xi_{k}$, computed in a similar fashion to equation (21) such that $\mathcal{A}=\sum_{k=1}^{n} \mathcal{A}_{k}$.

Equations (3), (18), (21) and (25) show that the flame shape, flame area and hence mean flame position depend on the acoustic wave amplitudes just upstream of the flame. These depend on the flame front location both at the current time and at some previous times (due to propagation delays). In conclusion, the acoustic wave amplitude ahead of the flame depends on the flame position, but the flame position depends on these wave amplitudes, thus the acoustic discontinuity location and the flame position are now coupled.

To overcome this problem, we know that waves $A$ and $B$, moving away from the discontinuity, are obtained directly from the jump equations, and are not part of the coupled problem (even if the location at which they are emitted is for now unknown). The amplitude of waves $C$ and $D$ as they arrive at the discontinuity requires more work. From equations (12) and (13), it follows that $C$ and $D$ at $x_{b}(t)$ depend only on the outgoing wave amplitudes $A$ and $B$ at previous times $t-\tau_{u}$ and $t-\tau_{d}$. Note that $\tau_{u}$ and $\tau_{d}$ now represent the times taken for a wave to travel from the discontinuity to the boundary and back to the changed position of the discontinuity again.

It is important to point out the times for which we write $\tau_{u_{A}}, \tau_{d_{B}}, \tau_{u_{C}}$ and $\tau_{d_{D}}$. Consider that we wish to observe the effects of incoming pressure waves on the flame induced discontinuity at a time $t$. We must know the values of the time delays $\tau_{u_{c}}$ and $\tau_{d_{D}}$ when waves $C$ and $B$ arrive at the discontinuity, i.e. at time $t$. In this case, it is clear that we must know the value of the time delays $\tau_{d_{B}}$ and $\tau_{u_{A}}$ when the waves $A$ and $B$ left said discontinuity; i.e. at time $t-\tau_{u}$ and $t-\tau_{d}$. This yields :

$$
\begin{aligned}
\tau_{u}(t) & =-\tau_{u_{A}}\left(x_{u}, t-\tau_{u}\right)-\tau_{u_{C}}\left(x_{u}, t\right) \\
\tau_{d}(t) & =\tau_{d_{B}}\left(x_{d}, t-\tau_{d}\right)+\tau_{d_{D}}\left(x_{d}, t\right)
\end{aligned}
$$

Using expressions (23) and (24), it is now clear that our above time delays, and therefore waves $C(t)$ 
and $D(t)$, depend on the 3 different positions of the flame induced discontinuity; namely $x_{b}\left(t-\tau_{u}\right)$, $x_{b}\left(t-\tau_{d}\right)$, and $x_{b}(t)$.

As is common in moving acoustic source problems, such as those encountered in helicopter acoustics (Morgans et al., 2005), the problem must be solved iteratively. The three coupled equations to be solved iteratively and simultaneously are:

$$
\begin{aligned}
\tau_{u} & =-\frac{x_{u}-x_{b}\left(t-\tau_{u}\right)}{\bar{c}_{u}-\bar{U}_{u}}-\frac{x-x_{b}(t)}{\bar{c}_{u}+\bar{U}_{u}} \\
\tau_{d} & =\frac{x_{d}-x_{b}\left(t-\tau_{d}\right)}{\bar{c}_{d}+\bar{U}_{d}}+\frac{x-x_{b}(t)}{\bar{c}_{d}-\bar{U}_{d}} \\
x_{b}(t)=x_{f}(t) & =\frac{\sum_{k=1}^{n} \mathcal{A}_{k}\left(t-\tau_{f}\right) \xi_{k}\left(t-\tau_{f}\right)}{\sum_{k=1}^{n} \mathcal{A}_{k}\left(t-\tau_{f}\right)}
\end{aligned}
$$

where $\mathcal{A}_{k}$ is obtained from the discrete version of $(21)$, and $\xi_{k}$ is obtained from the discrete version of (18). This system is readily solved using Newton-Raphson iterative methods.

\section{Results and discussion}

To investigate the effects of implementing the acoustic jump location more accurately, two sets of combustor test cases are considered. The first two cases are concerned with combustor stability, while the third considers limit cycle amplitude. For both sets, the upstream total temperature is $T_{0}=288 \mathrm{~K}$, mean heat release rate is $\bar{Q}=59 \mathrm{MJ} \mathrm{m}^{2} / \mathrm{s}$, downstream mean pressure is $\bar{P}=$ $1.013 \cdot 10^{5} \mathrm{~Pa}$ and duct dimensions are $r_{a}=1.75 \mathrm{~cm}$ and $r_{b}=3.5 \mathrm{~cm}$. We compare results for three discontinuity location implementations:

- the standard ducted-flame model with $x_{b}=0$;

- the discontinuity position fixed at the space-averaged steady flame position, $x_{b}=\bar{x}_{f}$; this is obtained from the steady form of equation (25), using the steady flame defined in (20);

- the discontinuity position moving in time to track the space-averaged flame position, $x_{b}(t)=$ $x_{f}(t)$.

The details of the test configurations are shown in Table 1.

In order to isolate the effect of time variation in the flame position, the flame time delay $\tau_{f}$ in the flame relation $Q(t) \propto \mathcal{A}\left(t-\tau_{f}\right)$ will be assumed constant here. It has previously been given as $\tau_{f}=\frac{0.42\left(x_{d}-x_{b}\right)}{u_{\text {gutter }}}$ (Wang and Dowling, 2005), implying a time varying flame time delay for cases in 
which $x_{b}$ varies with time.

[Table 1 about here.]

To obtain the results below, equation (18) is solved using a fourth order Runge-Kutta method for time integration, with time steps $\delta t=2,5 \cdot 10^{-} 5 \mathrm{~s}, \delta t=3 \cdot 10^{-} 5 \mathrm{~s}$ and $\delta t=1 \cdot 10^{-} 5 \mathrm{~s}$ for the three test cases respectively. A simple first order backward Euler method is used for spacial derivatives, using 64 points over a cross section of $1.75 \mathrm{~cm}$.

\subsection{Combustor stability}

In order to illustrate the effect that a time-varying flame position can have on combustor stability, test cases 1 and 2 are considered. The combustor geometries, upstream Mach number and boundary reflection coefficients are shown in Table 1.

The dominant oscillation frequencies for these test cases are shown in Table 2. It is seen that accounting for the shift in the spatial-mean position of the flame gives rise to a frequency shift of up to $14 \%$, due to the change in mode shape (the cold and hot proportions of the duct vary when the flame location is shifted).

[Table 2 about here.]

The envelopes of the corresponding pressure oscillations are shown in Figures 4(a) and 4(b), where the normalised acoustic pressure, $p_{r e f}$, at location $x_{r e f}$ is given. For both cases:

- $x_{b}=0$ gives rise to an unstable system which quickly saturates into a limit cycle.

- $x_{b}=\bar{x}_{f}$ gives rise to an unstable system with a much lower oscillation amplitude (still slowly growing - true limit cycle saturation has not yet occurred)

- $x_{b}(t)=x_{f}(t)$ gives rise to a stable system whose oscillation amplitude is slowly decaying.

Thus accounting for the movement of the flame (by varying the position at which the flame-induced discontinuity is assumed to occur) is seen to have a stabilising effect on the system, to the extent that it can render a combustor stable which, for a fixed flame position, is unstable. In the same way that time variation in the simple example of Section 3 was able to alter linear stability, we find that this is also occurring in these thermoacoustics simulations.

[Figure 4 about here.] 


\subsection{Limit cycle amplitude}

In order to illustrate the effect of time variation on limit cycle amplitude, for combustors where the system remains unstable both in the absence and presence of a time varying flame position, test case 3 considers high amplitude limit cycle oscillations. These give rise to large amplitude motion of the flame-induced discontinuity. The combustor parameters are summarised in Table 1.

The dominant oscillation frequency shown in Table 2 shows a significant shift depending on where the discontinuity is imposed. The corresponding pressure oscillations are shown in Figure 4(c). It can be observed that using $x_{b}(t)=x_{f}(t)$ leads to a substantial $38 \%$ decrease in negative peak amplitude when compared to the $x_{b}=\bar{x}_{f}$ case. Furthermore, the $x_{b}(t)=x_{f}(t)$ configuration leads to a pulsating limit cycle regime, where the peak of the pulse is obtained at $t \approx 0.89 \mathrm{~s}$ in Figure $4(\mathrm{c})$. This demonstrates that there are likely to be conditions under which accounting for flame movement, by changing the location of the acoustic discontinuity, is important in determining the limit cycle amplitude.

Accounting for the movement of the flame has an effect more generally, rather than just for the specific test cases chosen. To show this we consider a combustor with the same characteristics as in test case 3, but with downstream combustor lengths ranging from $0.7 \mathrm{~m}$ to $1.5 \mathrm{~m}$. The relative difference between the peak amplitudes of the $x_{b}=\bar{x}_{f}$ and $x_{b}(t)=x_{f}(t)$ configuration are shown in Figure 4(d). This confirms that the movement of the discontinuity can induce large changes in limit cycle amplitude across a range of conditions. Furthermore, it is clear that large limit cycle amplitude in the $x_{b}=\bar{x}_{f}$ configuration leads to a larger impact of the moving acoustic discontinuity on limit cycle amplitude.

\subsection{Rayleigh source term}

To further understanding of why applying the acoustic jump location more accurately affects both stability and limit cycle amplitudes, the well-known Rayleigh source term, $p_{a} Q^{\prime}$ (averaged over an oscillation cycle) is considered. It is known from the Rayleigh criterion (Rayleigh, 1878; Chu, 1964; Durox et al., 2009) that the larger this source term, the more likely it is to exceed loss terms and give rise to instability.

For the combustor stability test cases ( 1 and 2$)$, the time integrated source term product, $p_{a} Q^{\prime}$, is shown in Figures 5(a) and 5(c). The pressure $p_{a}$ in the source term is calculated from the average 
of the upstream and downstream acoustic pressure at the acoustic discontinuity. It can clearly be seen that changing the location of the flame-induced discontinuity alters this source term. In fact, it appears to generally be the case that accounting for flame movement about a given position, and therefore changing the position of the acoustic discontinuity with time, reduces the source term i.e. flame/acoustic discontinuity movement itself has a stabilising effect.

The phase difference (obtained from the Hilbert transform) between the pressure at the flame, $p_{a}$, and the heat release fluctuation, $Q^{\prime}$, are shown in Figures $5(\mathrm{~b})$ and $5(\mathrm{~d})$. For test case 1, the boundaries of the system are perfectly reflective (i.e. $R_{u}=R_{d}=-1$ ) meaning that all remaining boundary losses are due to the mean flow (Nicoud and Wierczorek, 2009). For this case, the phase difference shown in Figures 5(b) and with the $x_{b}=\bar{x}_{f}$ configuration oscillates around the upper phase instability limit as defined by the Rayleigh criterion: $\frac{\pi}{2}$. A phase value so close to the instability limit explains why this system is only just unstable. A similar argument can be made for test case 2, except that it suffers from small losses at the boundary not just associated to the mean flow. Therefore, for the $x_{b}=\bar{x}_{f}$ configuration to remain unstable, $p_{a}$ and $Q^{\prime}$ must be "more in phase" (the phase difference between $p_{a}$ and $Q^{\prime}$ now oscillates around $0.445 \pi$, as opposed to test case 1 where it oscillated around $0.497 \pi$ ), as is shown in Figure 5(d). Note that the oscillations of the phase relationship in the $x_{b}=\bar{x}_{f}$ configuration are due to the non-linear effects of the G-Equation (18) (Lieuwen, 2005).

For both test cases 1 and 2 in the $x_{b}(t)=x_{f}(t)$ configuration, the Rayleigh source terms remain positive even though the systems are stable. This can be explained by the losses in the system due to mean flow, and non reflective boundary conditions. Also, the higher initial values of the Rayleigh source term are due to higher amplitudes of oscillations after the initial perturbation. The effect of the moving discontinuity leads to greater amplitude oscillations of the phase relationship, which are not perfectly symmetric about the (previous) mean value. The higher amplitude of the phase oscillations in the $x_{b}(t)=x_{f}(t)$ configuration, along with their asymmetry, participate in the stabilisation of the system: the heat release and acoustic pressure are more "out of phase" overall.

[Figure 5 about here.]

For the limit cycle test case (case 3), the Rayleigh source term is shown in Figure 5(e). Looking first at the $x_{b}=\bar{x}_{f}$ configuration, we can see that settling into limit cycle oscillations occurs when the phase difference oscillations induce no change in system energy over one period. The moving acoustic discontinuity $x_{b}(t)=x_{f}(t)$, induces a stabilising effect, and therefore a faster onset of 
limit cycle saturation. It is interesting to see that the low frequency oscillations observed in the Rayleigh source term coincide with low frequency pulsations in the Rayleigh phase difference. As the phase pulsation amplitude increases well above $0.5 \pi$, the Rayleigh source term decreases, the pressure fluctuation amplitude decreases, and the motion of the flame becomes smaller. This leads to smaller variations of the phase difference between $p_{a}$ and $Q^{\prime}$, so that the phase now remains between $(-0.5 \pi, 0.5 \pi)$, destabilising the system and leading to an increase in the Rayleigh source term and the pressure fluctuating amplitude. This explains the pulsation that was observed on Figure 4(c).

In summary, the effect of applying the discontinuity in acoustic wave amplitude so as to represent the time variation of the flame location can have an important effect on both the thermoacoustic stability and the dominant frequency. Some of this is due to the shifting of the average proportions of hot and cold regions of the duct (i.e. applying the discontinuity at the correct time-space mean location of the flame). However, the time variation of the flame induced discontinuity also appears to be feeding into the thermoacoustic characteristics, with the possibility of it affecting the stability and limit cycle amplitude of the combustor.

\section{Adaptive control}

Adaptive controllers have previously been shown to stabilise the instabilities exhibited by anchored ducted flames (both modelled using the G-Equation and experimentally). Self-tuning regulator (STR) adaptive controllers, for example, have been found to achieve stabilisation well beyond the bounds for which Lyapunov theory guarantees stabilisation (Evesque et al., 2000, 2003; Dowling and Morgans, 2005; Morgans and Annaswamy, 2008). It is therefore interesting to investigate whether time variation in the acoustic discontinuity location poses any complications when implementing adaptive control of this form. Adaptive control will be applied by measuring the acoustic pressure in the tube at a point $x_{r e f}$ and injecting and modifying the acoustic velocity upstream of the combustor at position $x_{u}$. Modifying the acoustic velocity has been shown to have a similar effect on the equivalence ratio as modifying the fuel input mass flow rate to the system (Lieuwen and Zinn, 1998). 


\subsection{Self Tuning Regulator Algorithm}

We can define our combustor system with the plant $F(s)=\frac{p_{r e f}(s)}{i(s)}$ where $i(s)$ denotes the external perturbation to the system, and $s$ is the Laplace variable. We apply a controller $K(s)=\frac{v_{c}(s)}{p_{r e f}(s)}$, obtaining the closed loop block diagram shown in Figure 6 .

[Figure 6 about here.]

Choosing a simple lead-lag compensator as a controller structure, our controller transfer function can be written as

$$
K(s)=k_{1}(t) \frac{s+z_{c}}{s+z_{c}+k_{2}(t)}
$$

where $z_{c}$ determines our controller zero, and $k_{1}(t)$ and $k_{2}(t)$ are our adaptive coefficients whose time scale is much greater that the period of oscillation of the instability of $F(s)$ (Evesque et al., 2003). Assuming that our closed loop system has no right half plane zeroes (Evesque et al., 2003; Morgans and Annaswamy, 2008), and that its relative degree is smaller or equal to one (Narendra and Annaswamy, 2009; Evesque et al., 2000), then there exists a Lyapunov function which will ensure system stability of the equivalent linear system if the following update rules are used:

$$
\begin{aligned}
& \frac{d k_{1}(t)}{d t}=-g_{1} p_{\text {ref }}(t)^{2} \\
& \frac{d k_{2}(t)}{d t}=g_{2} p_{r e f}(t) V(t)
\end{aligned}
$$

where $g_{1}, g_{2}$ are the adaptation rates of the updates rules, chosen to ensure good controller coefficient convergence rates, and positive high frequency gains of the closed loop system (Illingworth and Morgans, 2010), and $\frac{\partial V}{\partial t}=-z_{c} V(t)+v_{c}(t)$.

\subsection{Adaptive control applied to the moving acoustic discontinuity model}

The STR control method proposed above is theoretically guaranteed to stabilise combustors obeying a set of general criteria. It has however been shown to provide stabilisation even when these criteria are not fully met (e.g. for systems with small time delays) (Evesque et al., 2000, 2003; Dowling 
and Morgans, 2005). We now wish to determine the performance of these STR controllers with the added complexity of a moving acoustic discontinuity.

The adaptive controller was applied to the test cases presented in section 5 . For test cases 1 and 2 , the system is already stable when $x_{b}(t)=x_{f}(t)$. The adaptive control in this case simply accelerates convergence. From Figure 7 we can see that the STR algorithm converged the adaptive control coefficients $k_{1}$ and $k_{2}$ to comparable values for both the $x_{b}=\bar{x}_{f}$ and $x_{b}(t)=x_{f}(t)$ configurations, and for all three test cases, with the greatest different appearing in test case 3 .

The small difference between the adaptive controller coefficients for the moving discontinuity and fixed discontinuity cases clearly shows that the moving acoustic discontinuity only has a very limited impact on the applicability of adaptive controllers to the system.

[Figure 7 about here.]

\section{Conclusion}

In anchored ducted flame models for combustion instability, the spatial extent of both the flame and its range of movement are typically small compared to the acoustic wavelength. Accounting for the effect of flame movement on thermoacoustic behaviour has thus previously been thought unnecessary. We have shown, in this paper, that this is not necessarily the case.

By allowing the flame "source" of acoustic waves to vary in time, so as to track the flame movement, we have investigated the effect of a moving flame front. The above problem further couples the acoustics and flame equation, and we have a "retarded-time" method for solving this coupled problem. We have shown that accounting for the moving flame front can alter both linear stability and (for unstable systems) the limit cycle amplitude. A moving flame front has so far only been seen to provide a stabilising effect, resulting in a reduction in the Rayleigh source term, compared to the "frozen" flame front case. Reassuringly, flame front movement is not seen to substantially affect the performance of Self-Tuning Regulator adaptive controllers for suppressing combustion instabilities.

\section{References}

Balasubramanian, K. and Sujith, R. I. 2008. Non-normality and nonlinearity in combustion-acoustic interaction in diffusion flames. J. Fluid Mech., 594, 29-57. 
Bessa, M. 2012. Perturbations of Mathieu Equations with Parametric Excitation of Large Period. Adv. Dyn. Syst. Appl., 7(1), 17-30.

Blumenthal, R. S., Subramanian, P., Sujith, R. I., and Polifke, W. 2013. Novel perspectives on the dynamics of premixed flames. Combust. Flame., 160(7), 1215-1224.

Candel, S. 2002. Combustion Dynamics and Control: Progress and Challenges. Proc. Combust. Inst., 29, 1-28.

Chu, B.-T. 1964. On the Energy Transfer to Small Disturbances in Fluid Flow. Acta Mech., 1(3), $215-234$.

Culick, F. E. C. 1988. Combustion Instabilities In Liquid-Fueled Propulsion Systems - An Overview. Advis. Gr. Aerosp. Res. Dev., 450, 1-73.

Dowling, A. P. 1995. The calculation of thermoacoustic oscillations. J. Sound Vib., 180(4), 557-581.

Dowling, A. P. 1997. Nonlinear self-excited oscillations of a ducted flame. J. Fluid Mech., 346, 271-290.

Dowling, A. P. 1999. A kinematic model of a ducted flame. J. Fluid Mech., 394, 51-72.

Dowling, A. P. and Morgans, A. S. 2005. Feedback Control of Combustion Oscillations. Annu. Rev. Fluid Mech., 37, 151-182.

Dowling, A. P. and Stow, S. R. 2003. Acoustic Analysis of Gas Turbine Combustors. J. Propuls. Power., 19(5), 751-764.

Dowling, A. P. and Williams, J. E. F. 1983. Sound and Sources of Sound. Ellis Horwood Limited.

Durox, D., Schuller, T., Noiray, N., Birbaud, A.-L., and Candel, S. 2009. Rayleigh criterion and acoustic energy balance in unconfined self-sustained oscillating flames. Combust. Flame., 156(1) , 106-119.

Evesque, S. 2000. Adaptive Control of Combustion Oscillations. PhD thesis, University of Cambridge.

Evesque, S., Dowling, A. P., and Annaswamy, A. M. 2000. Adaptive Algorithms for Control of Combustion. In NATO RTO/AVT Symposium on Active Control Technology for Enhanced Performance in Land, Air, and Sea Vehicles, Braunschweig, Germany. 
Evesque, S., Dowling, A. P., and Annaswamy, A. M. 2003. Self-tuning regulators for combustion oscillations. Proc. R. Soc., 459, 1709-1749.

Fleifil, M., Annaswamy, A. M., Ghoneim, Z. A., and Ghoniem, A. F. 1996. Response of a Laminar Premixed Flame to Flow Oscillations : A Kinematic Model and Thermoacoustic Instability Results. Combust. Flame., 106, 487-510.

Floquet, G. 1883. Sur les équations différentielles linéaires à coefficients périodiques. Annales scientifiques de l'ENS., 66(2), 47-88.

Ghose Choudhury, A. and Guha, P. 2014. Damped equations of Mathieu type. Appl. Math. Comput., 229, 85-93.

Goh, C. S. and Morgans, A. S. 2013. The Influence of Entropy Waves on the Thermoacoustic Stability of a Model Combustor. Combust. Sci. Technol., 185(2), 249-268.

Hemchandra, S., Peters, N., and Lieuwen, T. 2011. Heat release response of acoustically forced turbulent premixed flames-role of kinematic restoration. Proc. Combust. Inst., 33(1), 1609-1617.

Hield, P. A., Brear, M. J., and Jin, S. H. 2009. Thermoacoustic limit cycles in a premixed laboratory combustor with open and choked exits. Combust. Flame., 156(9), 1683-1697.

Illingworth, S. and Morgans, A. S. 2010. Adaptive Feedback Control of Combustion Instability in Annular Combustors. Combust. Sci. Technol., 182(2), 143-164.

Kabiraj, L. and Sujith, R. I. 2012. Nonlinear self-excited thermoacoustic oscillations: intermittency and flame blowout. J. Fluid Mech., 713, 376-397.

Kashinath, K., Hemchandra, S., and Juniper, M. P. 2013. Nonlinear thermoacoustics of ducted premixed flames: The influence of perturbation convection speed. Combust. Flame., 160, 28562865.

Kerstein, A. R., Ashurst, W. T., and Williams, F. A. 1988. Field equation for interface propagation in an unsteady homogenous flow field. Phys. Rev., 37(7), 2728-2731.

Langhorne, P. J. 1988. Reheat buzz: an acoustically coupled combustion instability. Part 1. Experiment. J. Fluid Mech., 193, 417-443.

Langhorne, P. J. 1988. Reheat buzz: an acoustically coupled combustion instability. Part 2. Theory. J. Fluid Mech., 193, 445-473. 
Lieuwen, T. 2003. Modeling Premixed Combustion - Acoustic Wave Interactions : A Review. J. Propuls. Power., 19(5), 765-781.

Lieuwen, T. 2005. Nonlinear kinematic response of premixed flames to harmonic velocity disturbances. Proc. Combust. Inst., 30(2), 1725-1732.

Lieuwen, T. and Zinn, B. T. 1998. The role of equivalence ratio oscillations in driving combustion instabilities in low NOx gas turbines. Symp. Inst. Combust., 27(2), 1809-1816.

Morgans, A. S. and Annaswamy, A. M. 2008. Adaptive Control of Combustion Instabilities for Combustion Systems with Right-Half Plane Zeros. Combust. Sci. Technol., 180(9), 1549-1571.

Morgans, A. S., Karabasov, S. A., and Dowling, A. P. 2005. Transonic Helicopter Noise. AIAA J., 43(7), 1512-1524.

Morgans, A. S. and Stow, S. R. 2007. Model-based control of combustion instabilities in annular combustors. Combust. Flame., 150, 380-399.

Narendra, K. and Annaswamy, A. M. 2009. Stable Adaptive Systems. Dover Publications Inc., New York, USA.

Nicoud, F. and Wierczorek, K. 2009. About the zero Mach number assumption in the calculation of thermoacoustic instabilities. Int. J. Spray Combust. Dyn., 1(1), 67-111.

Noiray, N., Durox, D., Schuller, T., and Candel, S. 2008. A unified framework for nonlinear combustion instability analysis based on the flame describing function. J. Fluid Mech., 615, $139-167$.

Peracchio, A. A. and W. M. Proscia. 1999. Nonlinear Heat-Release/Acoustic Model for Thermoacoustic Instability in Lean Premixed Combustors. J. Eng. Gas Turbines Power., 121(3), $415-421$.

Preetham, Santosh, H., and Lieuwen, T. 2008. Dynamics of Laminar Premixed Flames Forced by Harmonic Velocity Disturbances. J. Propuls. Power., 24(6), 1390-1402.

Preetham, Thumuluru, S. K., Santosh, H., and Lieuwen, T. 2010. Linear Response of Laminar Premixed Flames to Flow Oscillations: Unsteady Stretch Effects. J. Propuls. Power., 26(3), $524-532$. 
Preetham, S. H. and Lieuwen, T. C. 2007. Response of turbulent premixed flames to harmonic acoustic forcing. Proc. Combust. Inst., 31(1), 1427-1434.

Rayleigh, J. W. S. 1878. On the instability of jets. Proc. Lond. Math. Soc., 10, 4-13.

Rienstra, S. W. and Hirschberg, A. 2012. An Introduction to Acoustics. Technical Report January, Eindhoven University of Technology.

Schuller, T., Durox, D., and Candel, S. 2003. A unified model for the prediction of laminar flame transfer functions: comparisons between conical and V-flame dynamics. Combust. Flame., 134, $21-34$.

Shin, D.-H. and Lieuwen, T. 2013. Flame wrinkle destruction processes in harmonically forced, turbulent premixed flames. J. Fluid Mech., 721, 484-513.

Stow, S. R. and Dowling, A. P. 2009. A Time-Domain Network Model for Nonlinear Thermoacoustic Oscillations. J. Eng. Gas Turbines Power., 131(3).

Wang, C.-H. and Dowling, A. P. 2005. Actively tuned passive control of combustion instabilities. In Advances in Combustion and Noise Control, pages 45-64. Cranfield University Press.

Yuan, X., Glover, K., and Dowling, A. P. 2010. Modeling Investigation for Thermoacoustic Oscillation Control. In American Control Conference, Baltimore, USA. 


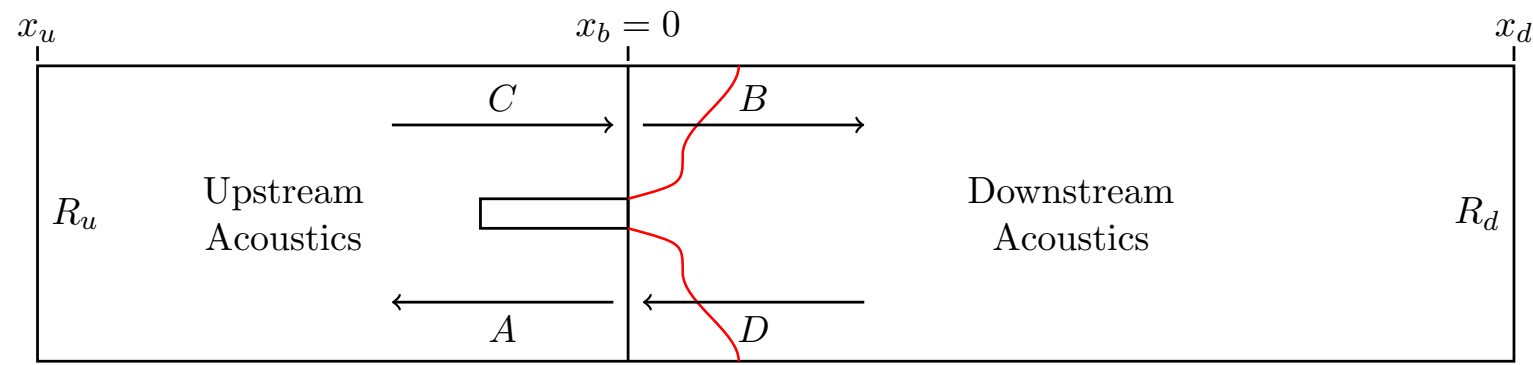

Figure 1: Schematic of the combustor duct, showing the incoming and outgoing pressure waves and the pressure reflection coefficients at the boundary. The discontinuity in acoustic wave amplitude is positioned at the point where the flame is anchored to the flame holder: $x_{b}=0$. 


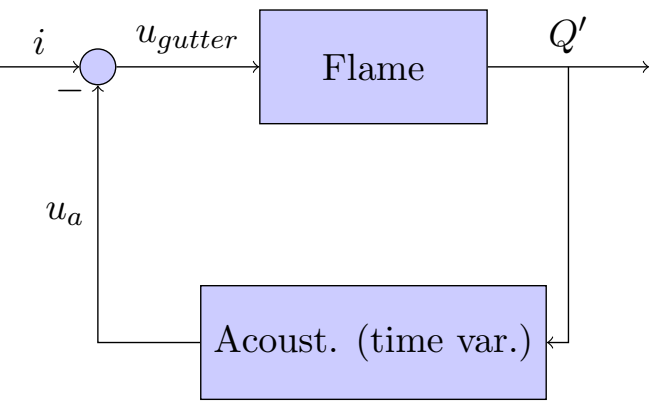

Figure 2: Block diagram of the ducted flame with time varying acoustics. $i$ denotes the external perturbation. 


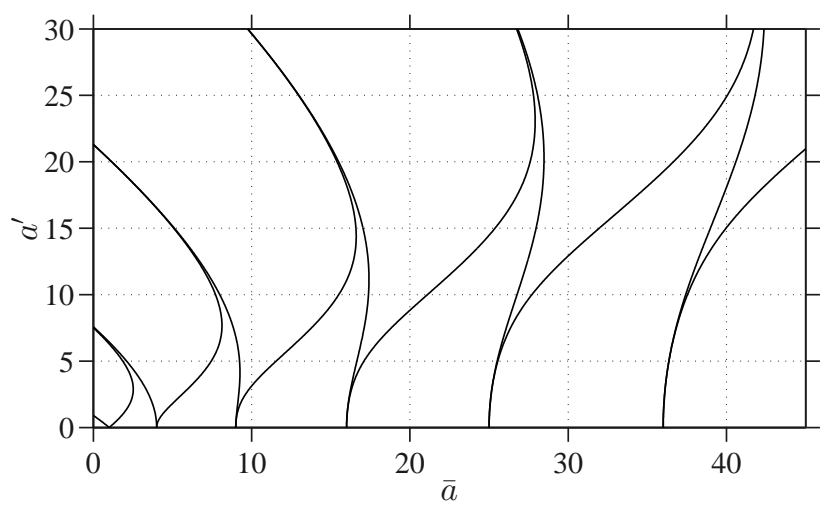

Figure 3: Ince-Strutt stability diagram of the Mathieu Equation. 


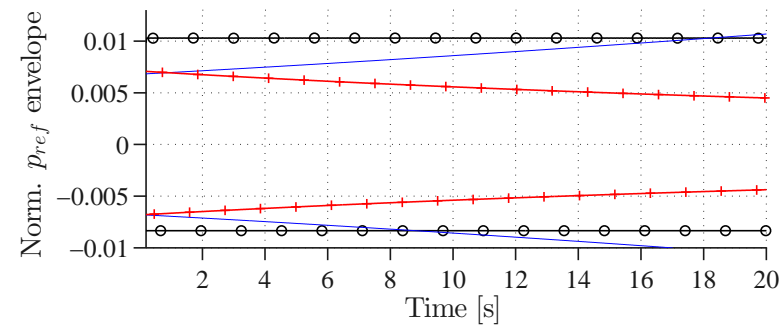

(a) Envelope of the normalised pressure $p_{\text {ref }}$ for test case 1: $\circ x_{b}=0$ (multiplied by a factor $\left.1 / 6\right) ;-x_{b}=\bar{x}_{f} ;+$ $x_{b}(t)=x_{f}(t)$.

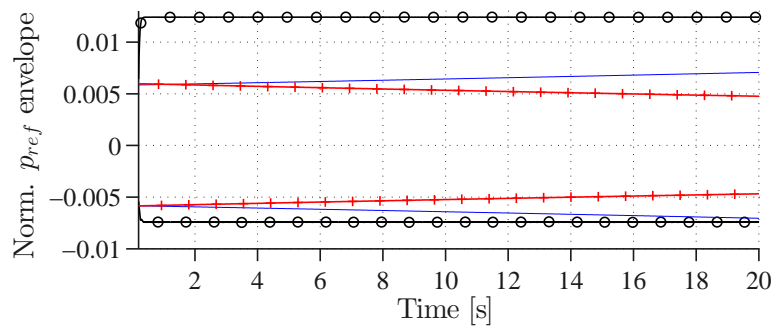

(b) Envelope of the normalised pressure $p_{\text {ref }}$ for test case 2: $\circ x_{b}=0$ (multiplied by a factor $1 / 5.6$ ); $-x_{b}=\bar{x}_{f}$; $+x_{b}(t)=x_{f}(t)$.

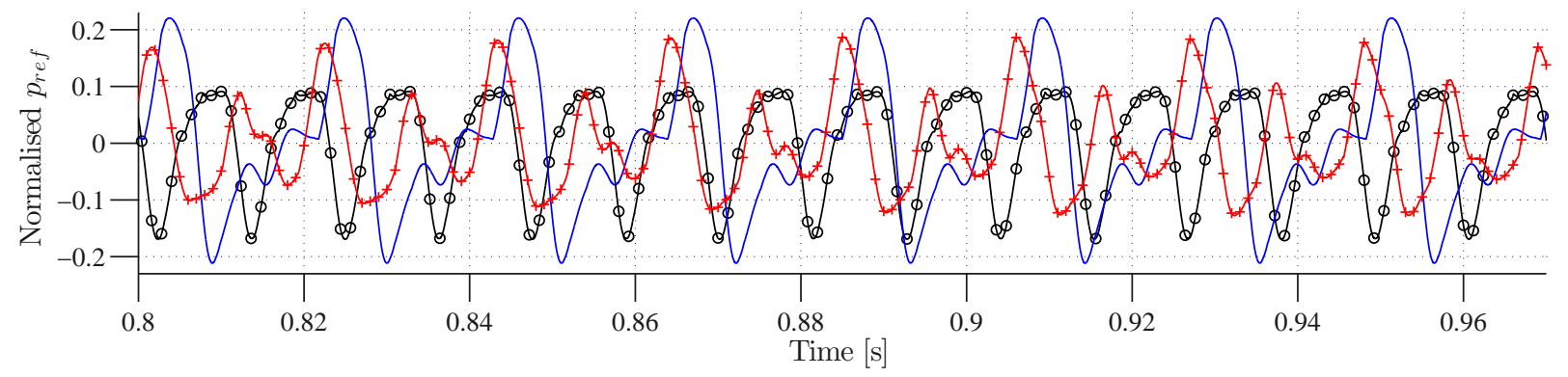

(c) Normalised limit cycle pressure fluctuation, $p_{\text {ref }}$ : $\circ x_{b}=0 ;-x_{b}=\bar{x}_{f} ;+x_{b}(t)=x_{f}(t)$

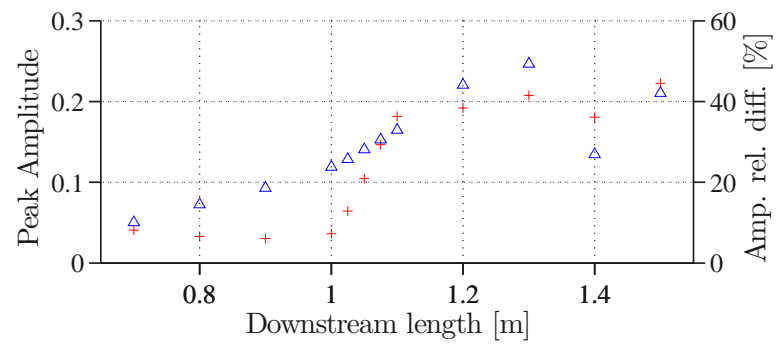

(d) Relative difference between the $x_{b}=\bar{x}_{f}$ and $x_{b}(t)=$ $x_{f}(t)$ model peak amplitudes $(+)$, and absolute value of the peak amplitude in the $x_{b}=\bar{x}_{f}$ configuration $(\triangle)$.

Figure 4: Pressure calculated with the new model and compared to standard anchored ducted flame results. 


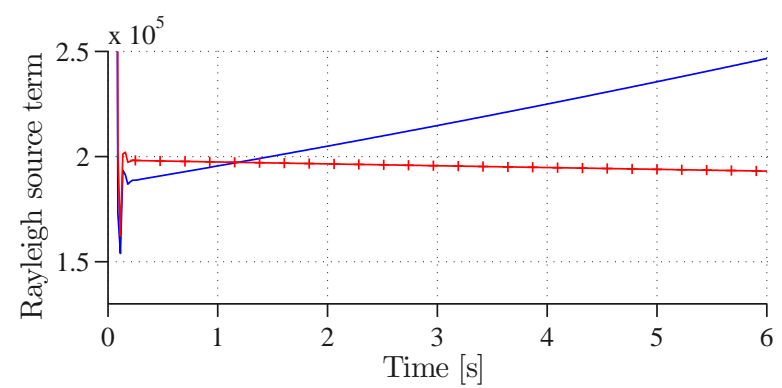

(a) Test Case 1: Rayleigh Source Term

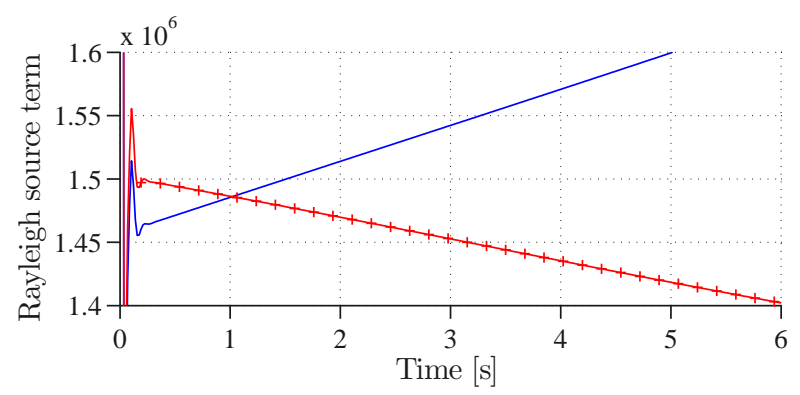

(c) Test Case 2: Rayleigh Source Term

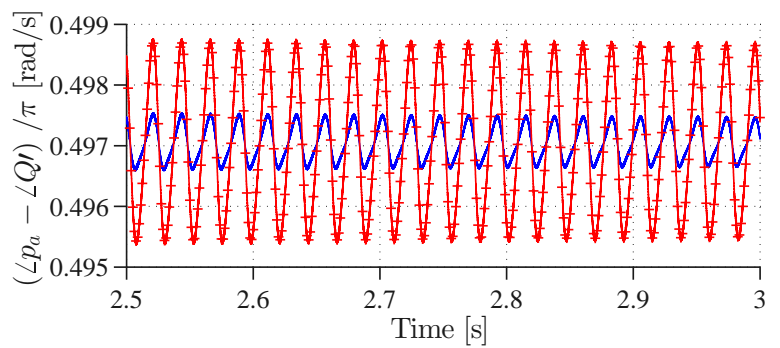

(b) Test Case 1: Phase difference between $p_{a}$ and $Q^{\prime}$

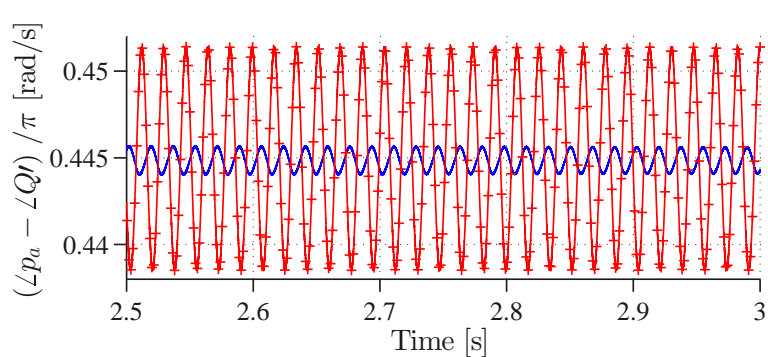

(d) Test Case 2: Phase difference between $p_{a}$ and $Q^{\prime}$

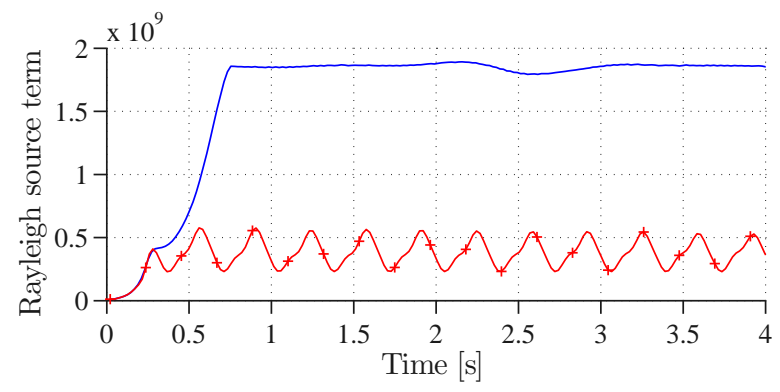

(e) Test Case 3: Rayleigh Source Term

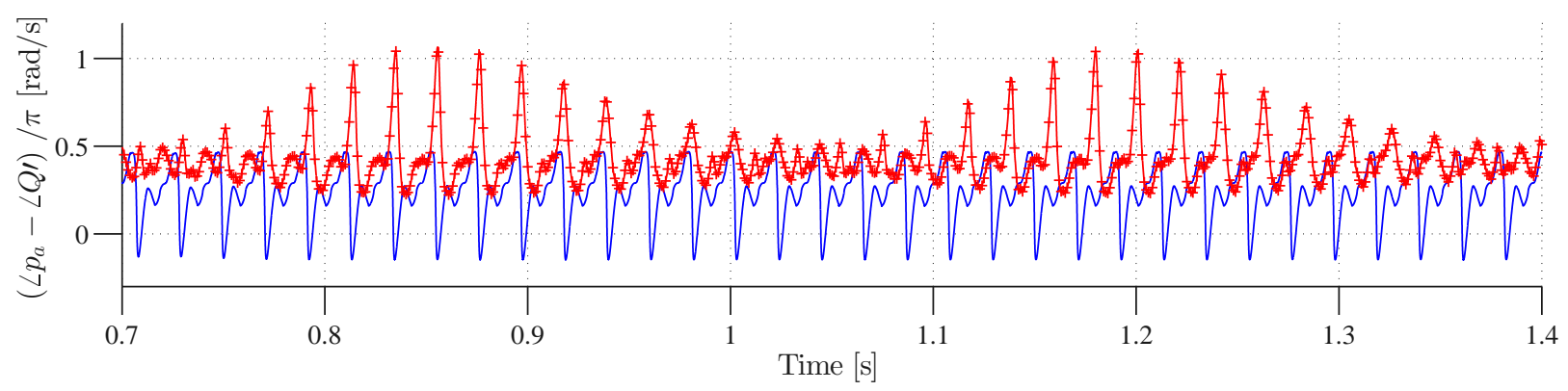

(f) Test Case 3: Phase difference between $p_{a}$ and $Q^{\prime}$

Figure 5: Rayleigh source term and phase difference between the pressure at the flame $p_{a}$ and $Q^{\prime}$ for different test cases: $-x_{b}=\bar{x}_{f} ;+x_{b}(t)=x_{f}(t)$. 


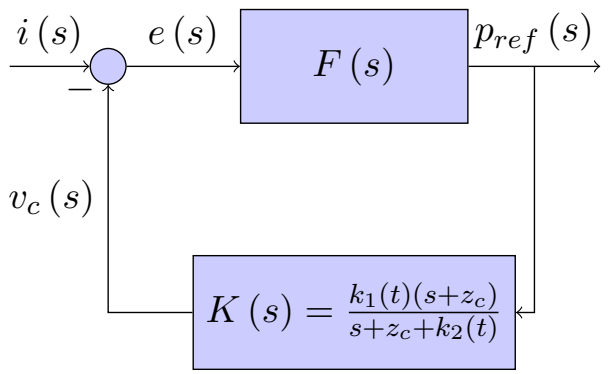

Figure 6: Block diagram of the sytem with an adaptive controller. 


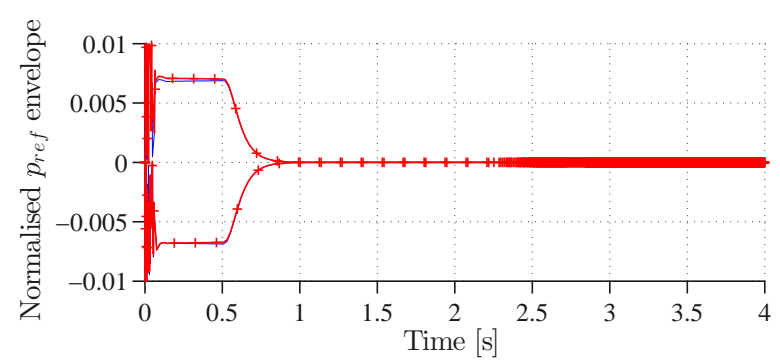

(a) Normalised acoustic pressure $p_{r e f}$, test case 1 .

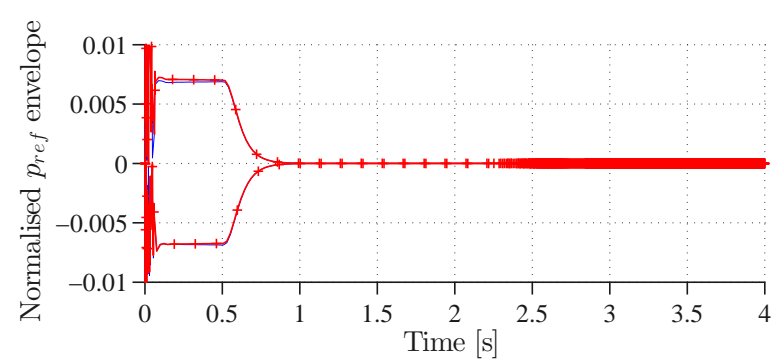

(c) Normalised acoustic pressure $p_{r e f}$, test case 2 .

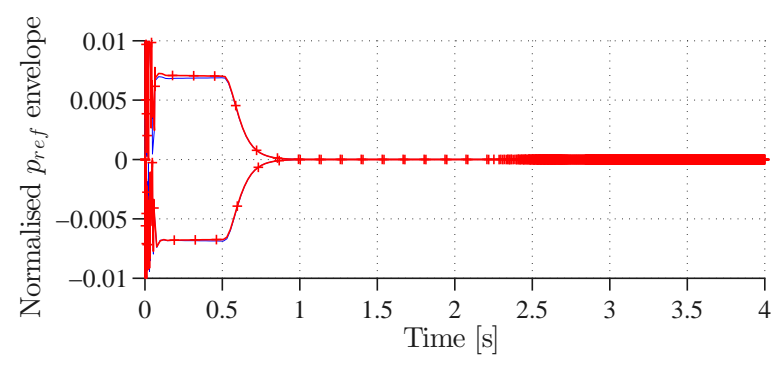

(e) Normalised acoustic pressure $p_{\text {ref }}$, test case 3 .

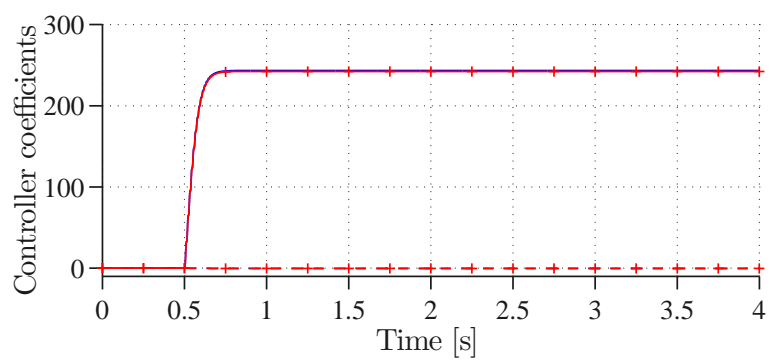

(b) Controller coefficients, test case 1; dashed lines indicate $k_{2}(t)$

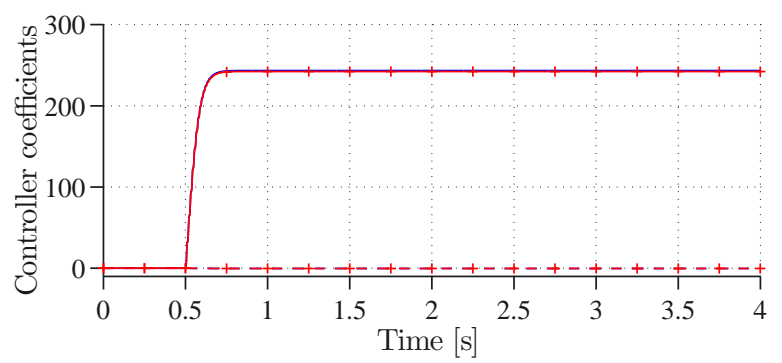

(d) Controller coefficients, test case 2; dashed lines indicate $k_{2}(t)$

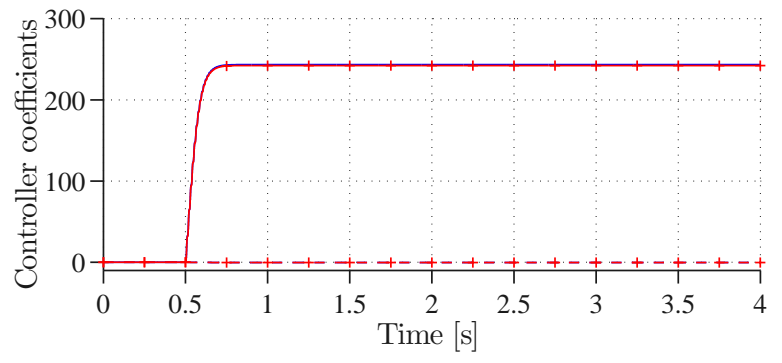

(f) Controller coefficients, test case 3; dashed lines indicate $k_{2}(t)$

Figure 7: Adaptive control results for test cases 1, 2 and $3 ;-x_{b}=\bar{x}_{f} ;+x_{b}(t)=x_{f}(t)$; 
Table 1: Combustor parameters used in test cases showing the effect of the moving acoustic discontinuity.

\begin{tabular}{|c|c|c|c|c|c|c|}
\hline Case & $x_{u}[\mathrm{~m}]$ & $x_{d}[\mathrm{~m}]$ & $x_{r e f}[\mathrm{~m}]$ & $R_{u}$ & $R_{d}$ & $M_{u}$ \\
\hline 1 & -0.3 & .841 & 0.33 & -1 & -1 & 0.08 \\
\hline 2 & -1.5 & .6855 & 0.22 & 0.85 & -.98 & 0.08 \\
\hline 3 & -1.0 & 1.2 & 0.33 & 0.85 & -.98 & 0.08 \\
\hline
\end{tabular}


Table 2: Combustor pressure dominant oscillation frequencies for the test cases investigating combustor stability and limit cycle amplitude.

\begin{tabular}{|c|c|c|c|}
\hline & \multicolumn{3}{|c|}{ Angular frequencies $[\mathrm{rad} / \mathrm{s}]$} \\
\hline Case & $x_{b}=0$ & $x_{b}=\bar{x}_{f}$ & $x_{b}(t)=x_{f}(t)$ \\
\hline 1 & 488 & 278 & 278 \\
\hline 2 & 335 & 359 & 359 \\
\hline 3 & 556 & 300 & 291 \\
\hline
\end{tabular}

